\title{
The Science and Detection of Tilting
}

\author{
Xingjie Wei ${ }^{\star}$ \\ Psychometrics Centre, \\ University of Cambridge \\ xw323@cam.ac.uk
}

\author{
Jussi Palomäki* \\ Cognitive Science Unit, \\ University of Helsinki \\ jussi.palomaki@helsinki.fi \\ Peter Robinson \\ Computer Laboratory, \\ University of Cambridge \\ pr10@cl.cam.ac.uk
}

\author{
Jeff Yan \\ School of Computing and \\ Communications, University of \\ Lancaster \\ jeff.yan@lancaster.ac.uk
}

\begin{abstract}
Tilting refers to losing control due to negative emotions, behaving erratically, and thereby suffering adverse consequences. The term tilt originated in poker playing communities, where it is often a consequence of so called bad beats, i.e. losing with an unlikely-to-lose poker hand. Often resulting in devastating monetary losses, tilting is ubiquitous and well known in poker, but rarely studied despite its significance. In this paper, we argue that tilting is a fertile topic for interdisciplinary research both for psychologists and computer scientists. Specifically, we propose to study the manifestation of tilting via facial emotion expressions, and motivate the development of an automatic tilt-detection system. The scientific understanding of the psychology of tilting can be increased via a computing approach, which has not been previously attempted. Automatic tilting detection will lead to a practical technology that reduces poker players' monetary losses and improves their well being through reduced tilting. We also argue that while tilting is best known as a poker phenomenon, it also exists in other contexts. Thus, the idea we suggest is a novel application of computer vision, affective computing and multimedia technologies in the real world, across many domains.
\end{abstract}

\section{Keywords}

Tilting; facial expression; poker;affective computing

\section{INTRODUCTION}

Poker is a popular game played by over a hundred million players worldwide, most notably online. The yearly market value of online poker is estimated in billions of US dollars [20]. Poker is also often featured in various blockbuster movies (James Bond, X-Men, etc.), and dozens of television

\section{*denotes equal contribution.}

Permission to make digital or hard copies of all or part of this work for personal or classroom use is granted without fee provided that copies are not made or distributed for profit or commercial advantage and that copies bear this notice and the full citation on the first page. Copyrights for components of this work owned by others than the author(s) must be honored. Abstracting with credit is permitted. To copy otherwise, or republish, to post on servers or to redistribute to lists, requires prior specific permission and/or a fee. Request permissions from permissions@acm.org.

ICMR'16, June 06 - 09, 2016, New York, NY, USA

(C) 2016 Copyright held by the owner/author(s). Publication rights licensed to ACM. ISBN 978-1-4503-4359-6/16/06 .. \$15.00

DOI: http://dx.doi.org/10.1145/2911996.2912019 programs are exclusively dedicated to it, which highlights its cultural significance - nearly everyone is familiar with terms like bluff and poker-face.

Poker involves constant decision-making and risk analysis with monetary stakes, and it inspired John von Neumann to invent game theory, which is the study of strategic cooperation and conflict between intelligent rational decisionmakers. In the recent years, poker has been used by researchers to study human decision-making processes [23], leading to interesting results and our increased scientific understanding of decision-making.

In poker, the term tilt refers to losing control and making detrimental, strategically weak decisions due to negative emotions, such as anger or frustration. Typical poker situations that cause tilt include, but are not limited to 1) losing in a situation where losing is perceivably highly improbable, for example, having a $90 \%$ chance of winning a big pot of money, but ending up losing it due to chance (or bad luck). This is known as encountering a bad beat in the poker community. 2) Prolonged series of losses (losing streaks), and 3) Factors external to the game mechanics, such as fatigue, or needling by other players (making provocative or stingy comments) [3]. Tilted poker players commonly adopt a sub-optimal and overly aggressive playing style, whereby they almost always end up losing superfluous amounts of money. This can result in losing one's entire life savings in a single 20-minute session [17].

It is well known in poker communities that tilting is a highly prevalent phenomenon. In a recent online study, within their last 6 months of poker playing, $88 \%$ of players reported having tilted severely at least once, $43 \%$ more than five times, and $24 \%$ more than 10 times [18]. This means that most poker players will eventually tilt, if they play long enough. It is thus not a stretch to state that tilting is the number one destroyer of bankrolls in poker.

Unfortunately, despite its significance, tilting has been rarely studied. There are only a few scientific studies on tilting in the psychology and gambling communities $[17,16$, 18]. These studies have analysed poker players' subjective self-reports and suggested that tilting - in addition to being related to the emotions of anger and frustration - is characterized by strong feelings of injustice. In other words, tilting seems to be a state of moral anger: losing due to bad luck is perceived as unfair, which motivates an aggressive retaliation strategy (in the name of justice), which, in turn, ends in disappointment in self, and rumination over 


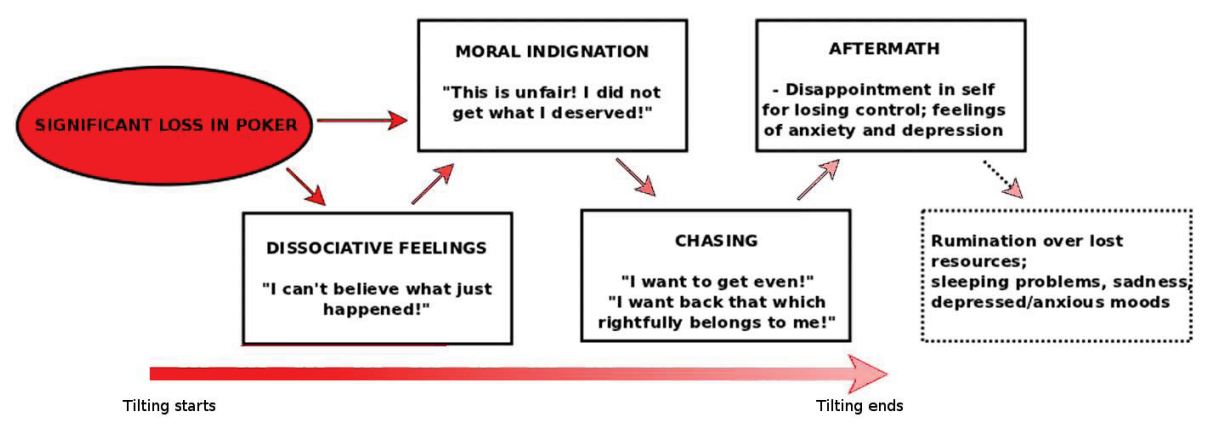

Figure 1: A model illustrating the phenomenology and aetiology of tilting. The small arrows indicate directions of causality. The bottom arrow indicates time.

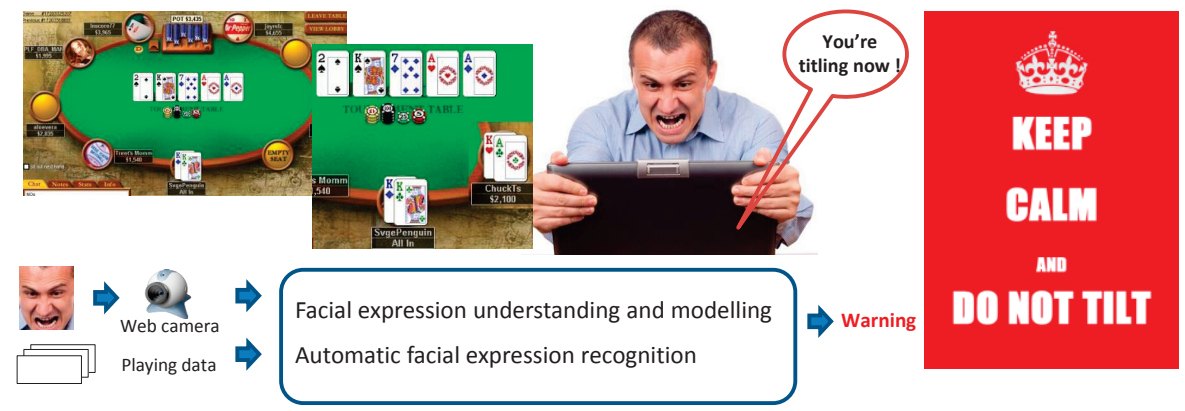

Figure 2: Illustration of poker tilting and automatic detection.

lost resources [16, 17]. This was a particularly common behavioural pattern in inexperienced players. The etiology and phenomenology of tilting is aptly depicted in a model formed by Palomäki, Laakasuo, and Salmela [17], which is based on a qualitative analysis of poker players' self-reported loss-induced emotions (see Figure 1).

It should also be noted that an increasing number of selfhelp books (e.g. [25]) have been published that specifically focus on how to deal with the emotional challenges posed by playing poker - i.e. the mental game of poker. At the heart of the challenges is tilting: each of these books recognises it as a remarkably significant single factor for players to lose excessive amounts of money. In other words, the mental game of poker is almost synonymous with tiltcontrol, and has become an increasingly pellucid subject matter especially during the era of online poker.

Interestingly, an increasing amount of anecdotal and empirical evidence suggests that tilting might in fact be a more universal human behavioural tendency. In a non-poker gambling context, behaviour similar to tilting is often seen in situations where people chase their losses [2]. This refers to people continuing to gamble only to win back what they had previously lost, for example, in slot machines, roulette or blackjack. As can be seen in Figure 1, chasing is also part of the aetiology of the tilting experience, although tilting as a whole is a more complex process.

Since tilting involves a strong moral anger component, it might also be related to road rage, which refers to aggressive or even retaliative behaviour in a motor vehicle. Road rage and tilting have a number of similarities, such as the aggressive and dangerous driving in road rage compared to the aggressive and detrimental betting behaviour in tilting, or the tendency to physically chase other motorists compared to the tendency of tilted players to chase their losses. Nonetheless, like tilting, road rage is often discussed colloquially but rarely studied empirically - at least not within a cohesive theoretical framework [5].

Moreover, even in the online communities of the popular Starcraft 2 game, the term tilted is often used to describe situations where players lose self-control and start playing overly aggressively and erratically. Finally, similar observations have also been made in tennis and golf [22].

Tilting is thus a significant and fascinating psychological phenomenon, and understanding it helps to better understand how emotions influence our behaviour and well-being. However, there seems to be no previous research on tilting in the computer science communities.

In this paper, we motivate the study of the psychology of tilting via a computing approach. Specifically, we propose to examine how tilting is manifested via facial emotion expressions. Methods and algorithms in computer vision, affective computing and multimedia will contribute to increasing the current scientific understanding of the psychology of tilting, a research goal that neither psychologists nor computer scientists would achieve alone. On the other hand, the understanding of how tilting is manifested via facial expressions will enlighten the development of an automatic tilt-detection system, which defines a novel application of computer vision and affective computing and multimedia technologies.

The overall illustration of our idea is shown in Figure 2. Since tilting is originally a poker phenomenon, we will employ poker as a tool to study it. A camera is used to capture videos of the poker players' facial expressions while 
they are playing. Quantitative data of the players' in-game poker actions (i.e. poker hand data) will be collected, and players will also keep a self-report diary to record their own experiences of tilting. Thereafter behaviour analysis and automatic facial expression recognition can be performed on these data. The results will form the basis of the automatic tilt detection system.

There have been no previous attempts to apply automatic facial expression analysis techniques to study the multifaceted and pervasive phenomenon of tilting. Such an interdisciplinary research project needs to draw heavily not only from theories of emotions and decision-making in cognitive psychology, but also from sophisticated techniques of facial expression recognition in computer science.

The rest of this paper is organised as follows. In Section 2 we introduce the theoretical foundations of emotions and facial expressions. In Section 3 we discuss related work. In Section 4 we discuss two important research questions in tilting and outline specific objectives for future research. In Section 5 we present an approach of combining behaviour analysis and facial expression recognition techniques to automatically detect the tilting behaviour. Finally, we conclude in Section 7 with a summary of the significance of our proposed idea, which extends beyond the context of poker.

\section{THEORETICAL FOUNDATIONS}

\subsection{Scientific theories of emotions}

It is clear that emotions are at the core of tilting. Emotions are multifaceted phenomena and studied within many different theoretical frameworks. Typically, emotion researchers are interested in the following questions: 1) what is the overall function of emotions (what they are for)? 2) what factors determine which emotion is experienced in a given situation; and 3) how do emotions relate to physiological processes in the body [7] ? There is some debate over whether emotions should be conceptualized as discrete or dimensional phenomena. A discrete model of emotions claims that different emotions correspond to specific physiological reactions and body expressions. According to discrete models, the physiological changes occurring in the body during experiences of anger are distinct from those occurring during experiences of fear. Dimensional models, in turn, argue that emotions are not so easily distinguished from one another at the physiological level, and that they should instead be defined according to one or more dimensions: for example, arousal (or activation; ranging from low to high), valence (ranging from unpleasant to pleasant), or incentive (ranging from withdrawal to approach) [21].

We focus briefly on two theoretical frameworks in emotion research: cognitive appraisal and evolutionary psychology theories of emotions. These frameworks are not mutually incompatible, but do differ in many key aspects.

According to cognitive appraisal theories [15], emotion elicitation depends on how various situations are appraised: two people can experience a very different emotion in an identical situation, if they appraise that situation differently. What is a compliment for some (nice hair!) may be a sarcastic insult for others. Appraisal is sometimes viewed as a cognitive process that is conceptually separate from emotional processes, as emotional reactions are assumed to follow cognitive appraisals. However, appraisals can also be unconscious, in which case the distinction between emotion and cognition becomes less obvious [15]. This framework has previously been adopted in computational modelling of human emotion [13].

However, appraisal theories of emotions are not really theories insofar as they mainly aim at describing how situational factors relate to emotion elicitation, but do not answer the deeper questions, such as "why do we have emotions?" or "what are emotions for"? The evolutionarypsychology theory of emotions is not so much concerned about the distinction between cognitive and emotional processes, but rather seeks to explain what the overall function or purpose of emotions is. In this paper, we adopt this view of emotions, as will be detailed below, because we believe it has the strongest empirical backing in both psychology and biology [31]. The evolutionary-psychologist theory also draws from both discrete and dimensional conceptualisations of emotions to create a uniform picture of what emotions really are.

\subsection{Emotions and facial expressions}

According to evolutionary psychologists, emotions are defined as coordinated psycho-physiological changes in the body and mind in response to specific (e.g. threatening or inviting) stimuli, which increased the fitness of our ancestors' genes.

For example, if we accidentally run into a bear while camping, we would freeze, our hearts would start pounding, and we would allocate all our attention towards resolving the threat. In other words, we would feel fear, which, in this situation, is a psycho-physiological manifestation of the so-called adaptive flight-or-flight response. We would at this moment also display fear on our faces, which is made possible by human facial muscles controlling our facial expressions. It is well known that discrete facial displays of emotions are a human universal, and the study of facial emotion expression dates back to Darwin in the 1800s. Putatively, displaying emotions on their faces made communication easier and faster for our ancestors in dangerous environments. The ability to effectively convey or interpret feelings enables quicker reactions in threatening situations. For example, a facial fear expression is a quicker way to signal danger than other more elaborative ways of communication, such as words.

The scientific study of universal facial emotions rose to general popularity in the 1970s, largely because of the crosscultural studies conducted by psychologist Paul Ekman [6]. However, the early studies were based on human observers and subjective evaluations of facial expressions, which are prone to errors of judgment and thus often unreliable. These limitations have since been overcome by representing facial expressions in terms of a fixed set of facial parameters, which can be calculated based on carefully observed movements in facial muscles. Currently, there is strong evidence for six basic emotions, that people across cultures display on their faces: joy/happiness, sadness, anger, fear, disgust and surprise (for an alternative view of four instead of six basic emotions, see [9]).

The term macro-expression is generally used to describe both the six basic facial emotions and other large-scale facial muscle movements. Unlike macro-expressions, microexpressions are brief (lasting less than 100ms), involuntary human facial expression associated with experiencing 
emotions. Micro-expressions typically occur in high-stakes situations, in which people have something significant to lose or gain - especially when the person does not consciously know what $\mathrm{s} /$ he is feeling, or is consciously trying to hide an emotion [6].

Facial expressions, both macro and micro, can be represented as a fixed set of parameters via the Facial Action Coding System (FACS). FACS defines atomic facial muscle actions as Action Unit numbers (AUs), which can describe almost any facial expression. For example, anger can be represented by the combined activation of AUs 4, 5, 7, 23 and 24 (the description of each AU is presented in Table 1).

Also more complex cognitive mental states such as bothered or disbelieving can be represented as facial expressions, but these are beyond the scope of the current paper.

\section{RELATED WORK}

As mentioned in Section 1, the only scientific studies on tilting were conducted by Palomäki and colleagues' $[17,16$, 18], which are useful in building a preliminary framework for understanding the psychology of tilting. However, these studies relied on poker players' subjective self-reports observations of actual tilting behaviour were not made. Furthermore, although other facial expressions have been studied in automatic facial expression recognition, there is no previous research on tilting in computer science communities.

Facial expression techniques have been widely employed in human-computer interaction applications. Despite having solid theoretical foundations, automatic micro-expression analysis techniques have not attracted much attention in the field of affective gaming (the study of how human affects and emotions can be recognised, interpreted and processed to enhance the gaming experience).

Compared with other methods used in studies of affective processes, such as measuring body movements or poses, or psycho-physiological emotion-related signals, such as heart beat rates, skin conductance responses, or electroencephalography, the vision based facial macro- and microexpression analysis system has unique advantages: 1) it requires only a cost-effective camera (even micro-expressions can be detected from videos with a frame-rate of $25 \mathrm{fps}$ and a resolution of $640 \times 480 ;[4,19]) ; 2)$ it is non-intrusive and easily adopted by poker players [10]

Analysing betting sequences and poker hand records (i.e. monitoring erratic betting behaviour by quantitative methods) could be useful for tilt-detection, but very difficult for current artificial intelligence (AI), although tremendous progress in poker AI research has been achieved by leading teams at the University of Alberta and Carnegie Mellon University [1]. Moreover, these teams have not specifically studied tilting at all.

In 2013, the Affective Computing Research Group led by Professor Rosalind Picard at the Massachusetts Institute of Technology were the first to collect naturalistic and spontaneous facial expressions in-the-wild via participants' cameras (at $14 \mathrm{fps}$ ), but they focused only on the emotion of joy [14]. Also in 2013, a leading team in facial expression analysis at the University of Oulu studied micro-expression detection during feelings of joy, sadness, disgust, fear and surprise in a laboratory setting; they were also the first to establish a spontaneous micro-expression database [10].

However, neither of these groups, nor any others, have evaluated the emotion of anger, which is highly relevant to tilting.

\section{RESEARCH QUESTIONS}

Previous evidence suggests that feelings of anger, frustration and injustice are implicated in tilting, but this knowledge is by no means sufficient to fully and objectively understand the phenomenon. Neither are the six basic emotions necessarily sufficient to fully characterise tilting behaviour. For example, it is not enough to merely look for facial displays of anger (or frustration) during poker play using pre-existing methods of facial expression classification, and, based on these, conclude that tilting had occurred. Various unknown macro- and/or micro-expressions might also be involved, for instance, those related to feelings of injustice (or some other still unascertained emotion). Furthermore, the exact temporal pattern/order of facial expressions during tilting has not been ascertained: do facial macro-expressions of frustration lead to micro-expressions of injustice, which, in turn, lead to macro-expressions of anger? Or does tilting begin with macro-expressions of anger (e.g. due to a bad beat), followed by micro-expressions of injustice and frustration? Is tilting more about short-lasting moral anger than prolonged feelings of frustration and/or anxiety, or vice versa? These questions are currently unanswered.

In other words, on a general level, we know how tilting feels (subjectively), but not what it actually looks like (objectively). We do not yet know what exactly are the objective facial expressions implicated in tilting behaviour. Thus, we propose to build on the existing anecdotal and empirical evidence on tilting by asking two subsequent simple but deep research questions:

- How does tilting manifest via facial expressions?

- Is this manifestation automatically detectable via computer vision methods?

People typically play online poker at home, facing a computer monitor, in a secluded environment. Since poker requires active concentration and task immersion, prominent facial expressions unrelated to the game are not likely to occur. Online poker is also played for real money and the stakes are often significant for many players. Arguably, while playing online, poker players do not benefit from attempting to hide their emotions (as they would in live poker). Micro-expressions are still likely to occur, given the emotional and monetary significance of the game to the players. We thus expect both macro- and micro-expressions to be implicated in online poker, and consequently in tilting.

We outline two specific objectives for future research:

1. Map the facial macro- and micro-expressions detected during actual tilting behaviour by employing facial expression analysis techniques.

2. Pioneer the development of an automatic system that detects expressions of tilting and warns players when tilting is imminent (Tilt-detector).

Previous studies of facial expression analysis have shown that detecting anger is more difficult than detecting other basic emotional expressions, such as surprise or joy. The display of anger requires a personal involvement, which is 


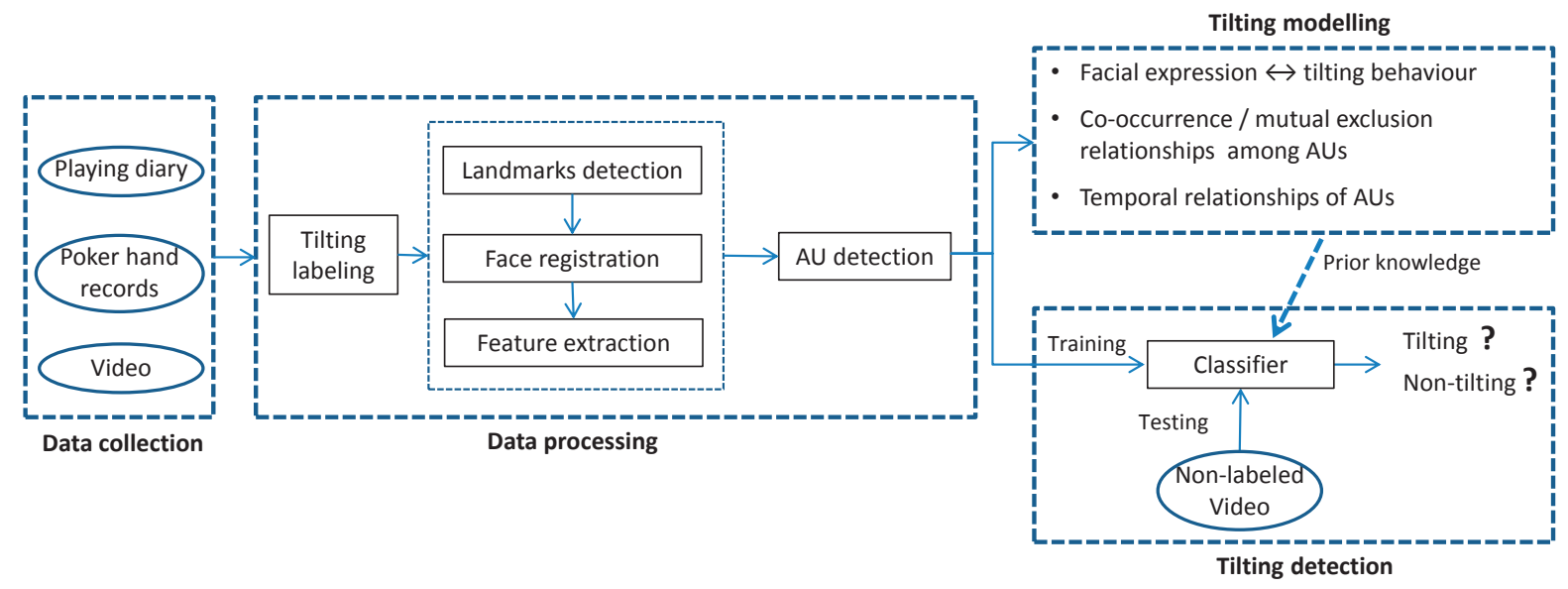

Figure 3: The framework of our research approach.

not easy to evoke using traditional methods of emotionelicitation. However, this is unlikely to be an issue in the current context, because we rely on the inherent nature of online poker as a naturalistic and emotional environment. This assumption has a strong empirical backing in psychology $[18]$.

\section{RESEARCH APPROACH}

The framework of our research approach is shown in Figure 3. It contains four main phases: data collection, data processing, tilting modelling and tilting detection. As mentioned in Section 1, the self-report playing diary, quantitative poker hand records and facial expression videos are first collected from the players. During data processing, the video frames are manually labelled for analysis (for the presence of tilting) according to the playing diary and hand record data, and then image processing is performed to extract features and detect AUs in these frames. The detected AUs are used for tilting modelling in three levels, from high level to low level.

In addition, the AUs and the previously extracted features are used to train classifiers for tilting detection, while the information acquired during the modelling process is considered in training. We explain each process in detail in the following sub-sections. Note that in each process, various specific techniques can be used in implementation our framework is flexible, extendable and not limited to the specific implementation methods introduced hereafter.

\subsection{Data collection}

For data collection, participants should be active poker players who play for real money and who have had personal experiences of tilting. Special care should be taken in screening for eligible participants. This includes, but is not limited to, assessing their level of poker experience, severity and frequency of self-reported tilting, and personality characteristics. Given that the overwhelming majority (about $95 \%$, see $[17,18]$ ) of poker players are male, female participants will not be included in the experiment due to unknown and possibly confounding gender effects. On the other hand, there is evidence [29] that cultural background affects nonverbal behaviour. To reduce unconstrained variability in the data, the data collection should only focus on western players. This enables performing a more effective analysis with less noisy data. However, we also note that our proposed framework is not necessarily limited to these constraints. Further research and development can be done without such constraints.

For a planned period of time, for example, 2-3 months, participants should be instructed to play as they would normally, with the exception of having their cameras on recording their facial expressions. Standard 25-fps webcams have yielded decent success for detecting micro-expressions, as evidenced in [10, 19]. Furthermore, Moore's law suggests that we will see better webcams widely deployed in the near future, which will further improve facial expression detection.

Participants should keep a playing diary, in which they record and time-stamp their experiences of tilting. These self-reports should include 1) perceived cause (e.g. bad beat), 2) exact time and duration, 3) perceived severity of the tilt, and 4) descriptions of the emotions felt.

Participants should also record their poker hand histories using poker tracking and analysis software such as Poker Tracker (www.pokertracker.com) or Hold'Em Manager (www.holdemmanager.com). These software import and parse poker hand histories from online poker sites and use this information to build a local database library. They thus allow for monitoring each participant's sessionby-session profit or loss, the number of hands played, and the amount of time played. Having detailed quantitative tracking records of the poker hands played will help participants accurately reflect on their playing sessions.

\subsection{Data processing}

\subsubsection{Tilting labelling}

After data collection, the video frames can be manually labelled as tilting, non-tilting and unknown, according to participants' self-reports and poker tracking data. For example, if a participant reports in his diary that during one session he started tilting around hand number 85 (the 85th played hand during that session) and stopped tilting around hand number 120 , we look at the recorded video frames between the time hands \#85 and \#120 were played, and label these frames as tilting. 


\subsubsection{Face data pre-processing}

For tilting and non-tilting video frames, a large number of facial landmarks, such as eye and eyebrow corners, nose tip and mouth corners, are automatically detected by the landmarks detector Face++ [32] in each frame. The face area is normalised according to its geometrical properties such as size and pose using geometrical or morphological transforms and then further normalised with respect to its photometrical properties such as illumination conditions. Thereafter the face area is cropped from the background and re-sized to a constant scale (i.e. $250 \times 250$ pixels), and appearance features such as the Local Binary Pattern (LBP) are extracted from a small region (i.e. $20 \times 20$ pixels) around each landmark. Finally, dimension reduction is performed using principal component analysis (PCA) to transform high-dimensional features into low-dimensional spaces for efficient classifier learning.

\subsubsection{AU detection}

To evaluate the subtle facial expressions related to tilting, AUs defined by the FACS are used to describe facial muscle actions. Typically, trained experts manually label AUs frame-by-frame from each video, which is time-consuming. In our framework, we adopt the automatic AU detectors BGCS [24] which includes a large number of trained AU models. Detailed information of AUs that can be detected is shown in Table 1.

Table 1: Description of AUs

\begin{tabular}{clcl}
\hline AU No. & Description & AU No. & Description \\
\hline 1 & Inner Brow Raiser & 17 & Chin Raiser \\
2 & Outer Brow Raiser & 18 & Lip Pucker \\
4 & Brow Lowerer & 20 & Lip Stretcher \\
5 & Upper Lid Raiser & 22 & Lip Funneler \\
6 & Cheek Raiser & 23 & Lip Tightener \\
7 & Lid Tightener & 24 & Lip Pressor \\
9 & Nose Wrinkler & 25 & Lips Part \\
10 & Upper Lip Raiser & 26 & Jaw Drop \\
11 & Nasolabial Deepener & 27 & Mouth Stretch \\
12 & Lip Corner Puller & 43 & Eyes Closed \\
14 & Dimpler & 45 & Blink \\
15 & Lip Corner Depressor & 46 & Wink \\
16 & Lower Lip depression & & \\
\hline
\end{tabular}

\subsection{Tilting modelling}

After the video frames have been labelled for the presence of tilt, automatic AU detection can be applied to these frames for tilting modelling. We create the models in three levels by: 1) building the mapping between tilting behaviour and facial expressions, 2) representing the co-occurrence / mutual exclusion relationships among AUs, and 3) creating models of the temporal relationships of AUs. The outputs of these three levels can tell us what tilting actually looks like and they can be used as prior knowledge to aid tilting detection.

Level 1: We first apply sequential regression analysis to assess which AUs are most related to the tilting expressions. Based on a small subset of discriminative AUs (whose frequencies among all the detected AUs exceed a specific threshold) and many iterations of regressions, an additional $\mathrm{AU}$ is added to the current subset if it increases the accuracy of predicting tilting expressions. After that, a tilting $A U$ set which contains a number of AUs of tilting expressions can be generated. Then we can contrast the tilting AU set with AUs detected from the non-tilt frames. By examining the differences in these two AU sets, we are able to answer whether or not tilting actually manifests as specific facial expression patterns.

In addition, we contrast the AUs of tilting expressions with the AUs of other basic facial expressions. As a hypothetical example, in the tilting frames, activity in AU4 (brow lowerer), AU5 (upper lid raiser), AU7 (lid tightener) and AU23 (lip tightener) might be pronounced. This reflects a facial expression akin to anger and perhaps disgust, which would be reasonable, given that tilting has been characterized in the literature as moral anger [17]. However, it should be noted that the expressions we might observe during tilting do not necessarily map directly into previously established emotion-related facial actions.

After these steps, we can see if the tilting expressions are a combination of other basic facial expressions (e.g. anger+sadness), or if new facial movements are involved. The modelling of Level 1 answers the first research question posed in Section 4.

Level 2: As shown in [24], some AUs usually co-occur to form meaningful expressions. For example, AU6 (cheek raiser) frequently co-occurs with AU12 (lip corner puller) and AU15 (lip corner depressor) when people smile. In contrast, some AUs almost never co-occur. For example, it is very difficult to activate AU24 (lip pressor) and AU25 (lips part) simultaneously.

The work in [27] shows that such co-occurrence and mutual exclusion relationships are important for facial action recognition. The pairwise co-occurrence probability of two AUs is computed as:

$$
P\left(A U_{i}=1 \mid A U_{j}=1\right)=\frac{N_{A U_{i}+A U_{j}}}{N_{A U_{j}}}
$$

where $N_{A U_{i}+A U_{j}}$ is the number of samples when $A U_{i}$ and $A U_{j}$ occur together and $N_{A U_{j}}$ is the number of samples of $A U_{j}$. Similarly, the pairwise mutual exclusion probability of two AUs is computed as:

$$
P\left(A U_{i}=0 \mid A U_{j}=0\right)=\frac{N_{\neg A U_{i}+\neg A U_{j}}}{N_{\neg A U_{j}}}
$$

where $N_{\neg A U_{i}+\neg A U_{j}}$ is the number of samples when neither $A U_{i}$ nor $A U_{j}$ occurs and $N_{\neg A U_{j}}$ is the number of sample when $A U_{j}$ does not occur.

We employ the Bayesian Networks (BN) to exploit the relationships among AUs detected in tilt frames. BN is a probabilistic model that represents a set of random variables and their conditional dependencies by a directed acyclic graph (DAG). Each AU is modelled as a node in a BN and the probabilistic dependencies among the AUs are characterised by the links between the nodes. The initial dependencies among the AUs are computed using equation (1) and (2) and a finer BN structure is learned by using structure learning methods [11].

Level 3: Besides the co-occurrence and mutual exclusion relationships, AUs also show strong temporal dependencies [26]. The activation of an AU can activate another AU (i.e. one is the cause and the other is the effect). For example, AU12 (lip corner puller) is usually followed by AU6 (cheek raiser) during smiling. 
The static BN in Level 2 captures the spatial dependencies among AUs. In order to represent the temporal relationships of AUs in tilting, we adopt the Dynamic Bayesian Network (DBN) [26], which consist of interconnected time slices of $\mathrm{BNs}$, and where the temporal relationships between two neighbours are modelled by a Hidden Makov Model (HMM). Therefore AUs can be regarded as a sequence of observations over time.

With models built in Level 2 and 3, we are able to better understand the relationships between AUs (i.e., facial micro features) and tilting expressions.

\subsection{Tilting detection}

After tilting modelling, we adopt facial expression recognition techniques to automatically detect the tilting expression from the videos. We use both the holistic appearance features (e.g., LBP features mentioned in Section 5.2.2) and AU features (See Table 1) for detection.

Baseline: We train a Support Vector Machine (SVM) using the LBP features extracted from tilting and non-tilting video frames as the baseline method, to detect the tilting expression as a whole.

AU based detection: The AU based detection focuses on facial micro features. As mentioned Section 5.3, a number of significant AUs related to tilting expressions are selected and used to train a SVM.

Intuitively, the second approach should generate superior performance to the baseline since it utilises high-level knowledge from the previously established associations between facial expressions and tilting behaviour. However, because the relationship between AUs and tilting expressions is still an open problem, the first approach, which detects tilt expression as a whole, could also prove useful.

\section{SIGNIFICANCE}

In this section, we summarise the significance of our idea and the proposed framework. Our framework is flexible and extensible, and can be applied to the study of other negative facial expressions.

\subsection{Authentic and spontaneous negative emo- tion data}

If data were successfully collected, they will be the first in the world on actual tilting behaviour, and on authentic and spontaneous negative emotional expressions, which are more difficult to obtain than positive expressions in naturalistic conditions. Currently, most of the public facial expression databases are limited to posed expressions collected under controlled laboratory conditions $[12,28]$. In contrast, the data we propose to collect will contain spontaneous and naturalistic (i.e. uncontrolled) expressions captured in realapplication scenarios. These expressions involve challenging elements, such as non-uniform illumination and changes in head poses. These data will be a valuable resource for researchers working in the domains of facial expression recognition, affective computing and psychology - the impact thus reaches far beyond the currently suggested research.

\subsection{Tilting prevention solution for poker}

An automatic tilt-detector can be developed based on the evaluation results of both the holistic and AU based approaches. The tilt-detector can be used to dissuade tilted players from playing. This can be done by a variety of means, including (but not limited to) force-closing or forcepausing the poker software, displaying warning-messages, or setting an upper limit to the number of online poker tables to play on. Moreover, very recently, the idea of studying the AUs and facial expressions simultaneously has attracted attention $[30,11]$ in the area of automatic facial recognition. We believe our proposed framework, which involves both the low-level (i.e. AUs) and high-level (i.e. tilting expressions) facial activities, will also contribute to the research in this area.

Many poker players strive to increase their skills and profits. This is reflected in the many poker self-help books focusing on the mental game aspects of the game, and also in the increased popularity of commercial online poker training sites such as www.deucescracked.com or www.pokersavvy.com, which offer downloadable training videos as well as hands-on coaching from professional poker players. Although tilting is ubiquitous and generally recognised by poker players as the most significant single reason for superfluous losses, there are currently no efficient software-based solutions to reduce tilting. Thus, the suggested research has also tremendous commercial potential. For example, an automatic tilt-detection system can be deployed as a cloud-based software-as-a-service. Poker players need only to switch on their webcam, and let the service do the rest.

Tilting less in poker equals losing much less money, or winning much more. More importantly, for tens of millions of poker players around the world, reduced tilting will undoubtedly result in increased well-being.

\subsection{Applications in other contexts}

Given that other tilt-like phenomena besides poker tilting exist in various settings - such as other types of gambling or games, road rage, or even sports - the detection of tiltrelated facial expressions has wide potential applicability. Casinos will not have a monetary incentive to deter their customers from tilting. However, in terms of responsible gambling, casinos are liable to ensure their customers' general welfare. This means casinos would not have reasonable moral grounds not to implement an effective means to reduce their customers' detrimental gambling behaviour (tilting). Likewise, inside a motor vehicle, it would be relatively easy to install computerized devices that automatically detect road rage (i.e. an aggressive behavioural tilt-like expression) and deter drivers from acting out. This application of affective computing could directly impact people's well being by saving lives.

Moreover, since poker is essentially a game of rapid financial investments, it closely resembles active stock trading. Like poker players, online stock traders often need to make multiple financial decisions over short timescales, and such decisions are adversely influenced by emotions. To our knowledge, there is only one pre-existing commercial device designed to reduce emotional arousal during online stock trading: The (Philips) Rationalizer, which measures online stock traders' heartbeat variability via a wristband and warns them if they get too excited or stressed (as indicated by increased heartbeat rates).

The most obvious caveat of the Rationalizer is its inability to differentiate between positive and negative emotions. For example, feeling enthusiastic or angry (positive an negative emotional states with high arousal, respectively) would 
both be reflected in an increased heartbeat rate and thus flagged by the Rationalizer as potentially "detrimental" for decision-making. Our proposed tilt-detector system does not have this limitation and could thus be easily utilized in an online stock-trading context. Moreover, our system is likely much more cost-effective than the Rationalizer, as the only hardware required is a standard web-camera.

It should also be noted that an early detection of microexpressions, including those related to tilting, has many potential applications in fields ranging from video security (e.g. surveillance), human-computer-interaction (e.g. robotics) to affective computing (e.g. gaming). Compared with the traditional facial expression recognition, early detection is a relatively unexplored problem [8].

The co-occurrence and mutual exclusion relationships among AUs modelled in Section 5.3 suggest that the presence of one AU can act as a strong prior for the presence or non-presence of others in tilting. In addition, the temporal relationships of tilting AUs enable tilting to be recognised from a sequence of observations over time. This information is helpful for building further models for early detection of tilting. This can be an interesting future research direction.

\section{SUMMARY}

In this paper, using online poker as a case study, we have motivated the study of the science and detection of tilting and related psychological phenomena. We have proposed a framework for examining how tilting is manifested via facial emotion expressions, and introduced a tilt-detection system based on behaviour analysis and automatic facial expression recognition techniques. On the one hand, the research idea discussed here would definitely produce new knowledge concerning emotion elicitation and facial expressions, e.g. during playing online poker in a very naturalistic setting, thus advancing the scientific understanding of the psychology of emotions and tilting - a significant but under-explored topic. On the other hand, the automatic tilt-detection introduced here defines a new problem for computer vision and affective computing, which is an interesting research area by itself, and can motivate novel applications in the future.

\section{REFERENCES}

[1] N. Bard, J. Hawkin, J. Rubin, and M. Zinkevich. The annual computer poker competition. AI Magazine, 34(2):112, 2013.

[2] R. B. Breen and M. Zuckerman. 'Chasing' in gambling behavior: personality and cognitive determinants. Pers. Indiv. Differ., 27(6):1097-1111, 1999.

[3] B. R. Browne. Going on tilt: Frequent poker players and control. J. Gambl. Behav., 5(1):3-21, 1989.

[4] W.-S. Chu, F. De la Torre, and J. Cohn. Selective transfer machine for personalized facial action unit detection. In $I E E E$ Conf. Computer Vision and Pattern Recognition (CVPR), pages 3515-3522, Jun. 2013.

[5] C. S. Dula and E. Geller. Risky, aggressive, or emotional driving: Addressing the need for consistent communication in research. J. Safety Res., 34(5):559-566, 2003.

[6] P. Ekman. Emotions Revealed: Recognizing Faces and Feelings to Improve Communication and Emotional Life. Henry Holt and Co., Apr. 2003.

[7] E. Fox. Emotion science cognitive and neuroscientific approaches to understanding human emotions. Palgrave Macmillan, 2008.

[8] M. Hoai and F. De la Torre. Max-margin early event detectors. Int. J. Comput. Vision, 107(2):191-202, 2014.

[9] R. E. Jack, O. G. Garrod, and P. G. Schyns. Dynamic facial expressions of emotion transmit an evolving hierarchy of signals over time. Curr. Biol., 24(2):187-192, 2014.
[10] X. Li, T. Pfister, X. Huang, G. Zhao, and M. Pietikainen. A spontaneous micro-expression database: Inducement, collection and baseline. In IEEE Int. Conf. Automatic Face and Gesture Recognition (FG), pages 1-6, Apr. 2013.

[11] Y. Li, S. Wang, Y. Zhao, and Q. Ji. Simultaneous facial feature tracking and facial expression recognition. IEEE Trans. Image Process., 22(7):2559-2573, Jul. 2013.

[12] P. Lucey, J. Cohn, T. Kanade, J. Saragih, Z. Ambadar, and I. Matthews. The extended cohn-kanade dataset $(\mathrm{CK}+)$ : A complete dataset for action unit and emotion-specified expression. In IEEE Conf. Computer Vision and Pattern Recognition Workshops (CVPRW), pages 94-101, Jun. 2010.

[13] S. Marsella and J. Gratch. Computationally modeling human emotion. Commun. ACM, 57(12):56-67, Nov 2014.

[14] D. McDuff, R. El Kaliouby, T. Senechal, M. Amr, J. Cohn, and R. Picard. Affectiva-MIT facial expression dataset (AM-FED): Naturalistic and spontaneous facial expressions collected in-the-wild;. In IEEE Conf. Computer Vision and Pattern Recognition Workshops (CVPRW), pages 881-888, Jun. 2013.

[15] A. Moors, P. C. Ellsworth, K. R. Scherer, and N. H. Frijda Appraisal theories of emotion: State of the art and future development. Emotion Review, 5(2):119-124, 2013.

[16] J. Palomäki, M. Laakasuo, and M. Salmela. "Don't worry, it's just poker!'-experience, self-rumination and self-reflection as determinants of decision-making in on-line poker. J. Gambl. Stud., 29(3):491-505, 2013.

[17] J. Palomäki, M. Laakasuo, and M. Salmela. "This is just so unfair!": A qualitative analysis of loss-induced emotions and tilting in on-line poker. Int. Gambl. Stud., 13(2):255-270, 2013

[18] J. Palomäki, M. Laakasuo, and M. Salmela. Losing more by losing it: Poker experience, sensitivity to losses and tilting severity. J. Gambl. Stud., 30(1):187-200, 2014.

[19] T. Pfister, X. Li, G. Zhao, and M. Pietikainen. Recognising spontaneous facial micro-expressions. In IEEE Int. Conf. Computer Vision (ICCV), pages 1449-1456, Nov 2011.

[20] K. Philander and I. Fiedler. Online poker in north america: Empirical evidence on its complementary effect on the offline gambling market. Gaming Law Review and Economics, 16(7-8):415-423, 2012.

[21] N. A. Remington, L. R. Fabrigar, and P. S. Visser. Reexamining the circumplex model of affect. J. Pers. Soc. Psychol., 79(2):286, 2000.

[22] B. Rotella. Golf is not a game of perfect. Simon and Schuster New York, NY 10020 USA, 1996

[23] E. J. Schlicht, S. Shimojo, C. F. Camerer, P. Battaglia, and K. Nakayama. Human wagering behavior depends on opponents' faces. PloS one, 5(7):e11663, 2010.

[24] Y. Song, D. McDuff, D. Vasisht, and A. Kapoor. Exploiting sparsity and co-occurrence structure for action unit recognition. In IEEE Int. Conf. Automatic Face and Gesture Recognition ( $F G)$, May 2015.

[25] J. Tendler and B. Carter. The mental game of poker: Proven strategies for improving tilt control, confidence, motivation, coping with variance, and more. Jared Tendler LLC, 2011.

[26] Y. Tong, J. Chen, and Q. Ji. A unified probabilistic framework for spontaneous facial action modeling and understanding. IEEE Trans. Pattern Anal. Mach. Intell., 32(2):258-273, Feb. 2010.

[27] Y. Tong, W. Liao, and Q. Ji. Facial action unit recognition by exploiting their dynamic and semantic relationships. IEEE Trans. Pattern Anal. Mach. Intell., 29(10):1683-1699, Oct. 2007.

[28] M. Valstar and M. Pantic. Induced disgust, happiness and surprise: an addition to the $\mathrm{mmi}$ facial expression database. In Int. Conf. Language Resources and Evaluation, Workshops on EMOTION, pages 65-70, 2010.

[29] A. Vrij and F. W. Winkel. Cultural patterns in dutch and surinam nonverbal behavior: An analysis of simulated police/citizen encounters. J. Nonverbal Behav., 15(3):169-184, 1991

[30] J. Wang, S. Wang, M. Huai, C. Wu, Z. Gao, Y. Liu, and Q. Ji. Capture expression-dependent au relations for expression recognition. In IEEE Int. Conf. Multimedia and Expo Workshops (ICMEW), pages 1-6, Jul. 2014.

[31] L. Workman and W. Reader. Evolutionary psychology. Cambridge University Press, 2014

[32] E. Zhou, H. Fan, Z. Cao, Y. Jiang, and Q. Yin. Extensive facial landmark localization with coarse-to-fine convolutional network cascade. In IEEE Int. Conf. Computer Vision Workshops (ICCVW), pages 386-391, Dec. 2013. 